\title{
MECHANISMS OF NONLETHAL PREDATOR EFFECT ON COHORT SIZE VARIATION: ECOLOGICAL AND EVOLUTIONARY IMPLICATIONS
}

\author{
Scott D. Peacor, ${ }^{1,2,5}$ Luis Schiesari, ${ }^{3,4}$ and Earl E. Werner ${ }^{3}$ \\ ${ }^{1}$ Department of Fisheries and Wildlife, Michigan State University, East Lansing, Michigan 48824 USA \\ ${ }^{2}$ NOAA Great Lakes Environmental Research Laboratory, 2205 Commonwealth Boulevard, Ann Arbor, Michigan 48105 USA \\ ${ }^{3}$ Department of Ecology and Evolutionary Biology, University of Michigan, Ann Arbor, Michigan 48109 USA \\ ${ }^{4}$ Environmental Management, School of Arts, Sciences, and Humanities, University of São Paulo, Av. Arlindo Bétio 1000, \\ São Paulo 03828-080 Brazil
}

\begin{abstract}
Understanding the factors responsible for generating size variation in cohorts of organisms is important for predicting their population and evolutionary dynamics. We group these factors into two broad classes: those due to scaling relationships between growth and size (size-dependent factors), and those due to individual trait differences other than size (sizeindependent factors; e.g., morphology, behavior, etc.). We develop a framework predicting that the nonlethal presence of predators can have a strong effect on size variation, the magnitude and sign of which depend on the relative influence of both factors. We present experimental results showing that size-independent factors can strongly contribute to size variation in anuran larvae, and that the presence of a larval dragonfly predator reduced expression of these size-independent factors. Further, a review of a number of experiments shows that the effect of this predator on relative size variation of a cohort ranged from negative at low growth rates to positive at high growth rates. At high growth rates, effects of size-dependent factors predominate, and predator presence causes an increase in the scaling of growth rate with size (larger individuals respond less strongly to predator presence than small individuals). Thus predator presence led to an increase in size variation. In contrast, at low growth rates, size-independent factors were relatively more important, and predator presence reduced expression of these size-independent factors. Consequently, predator presence led to a decrease in size variation. Our results therefore indicate a further mechanism whereby nonlethal predator effects can be manifest on prey species performance. These results have strong implications for both ecological and evolutionary processes. Theoretical studies indicate that changes in cohort size variation can have profound effects on population dynamics and stability, and therefore the mere presence of a predator could have important ecological consequences. Further, changes in cohort size variation can have important evolutionary implications through changes in trait heritability.
\end{abstract}

Key words: anuran; cohort; growth autocorrelation; growth rate; heritability; induction; nonconsumptive effect; nonlethal effect; phenotypic plasticity; predator; selection; size variation.

\section{INTRODUCTION}

Ecological and evolutionary biologists have long recognized that body size influences virtually every aspect of the relations between an organism and its internal and external environments. For example, size constrains or influences physiological rates, demographic parameters, the resources available or profitable, and vulnerability to predators (Peters 1983, Sebens 1987). In essence, size is a critical trait determining a species' niche (Hutchinson 1959, Werner and Gilliam 1984, Bonner 2006). As a consequence of the large import of size on the ecology of an organism, size changes that occur over ontogeny and the associated development of cohort size variation have important implications to species' per-

Manuscript received 22 June 2006; revised 2 November 2006; accepted 3 November 2006. Corresponding Editor: D. I. Bolnick.

${ }^{5}$ E-mail: peacor@msu.edu formance and natural selection. For example, there is considerable evidence that individual variation in size can affect population density and dynamics (e.g., Lomnicki 1988, DeAngelis et al. 1993, Uchmański and Grimm 1996, Wilson 1998, Uchmański 1999, De Roos et al. 2003). Variation in size may also reflect genetic differences that underlie selection (Van Valen 1965, Whitlock 1995, Conover and Munch 2002, Gardmark et al. 2003). Therefore factors that affect cohort size variation are implicated in exposing different traits to selection, or masking this variation from selection by preventing expression of trait characters.

Despite the critical importance of variation in cohort size structure to both ecological and evolutionary phenomena, we have little understanding of how size variation is generated (Uchmański 1985, Lomnicki 1988, Pfister and Stevens 2002). In particular, we know little about the relative contribution of two fundamentally different mechanisms that affect size variation. First, variation in genetic makeup or experience of organisms 
can cause and amplify size variation during growth over ontogeny. Second, variation in size, initially caused by trait differences or stochastic environmental effects, can be amplified or reduced due to the scaling effects of size on the growth process (see Predictions: Background). This distinction is important for conceptualizing and modeling the influences of size variation and determining how the environment affects size variation. Further, factors such as resource availability or environmental stress could modify the relative importance of sizedependent and size-independent processes, causing the predicted impact of a single factor to depend on context.

In this paper we explore the mechanisms responsible for the development of size variation in larval anuran cohorts. Specifically, we develop a verbal model based on the two broad processes that can affect size variation (trait variation or scaling effects), which predicts that predator presence can have a positive or negative effect on size variation, depending on mean growth rate of the cohort. In order to test these predictions, we first ask whether inherent among-individual trait differences that affect growth are large enough to strongly influence the development of size variation. Further, we address whether the presence of a common environmental stressor, risk of predation (i.e., "nonlethal" or "nonconsumptive" effects due to induced changes in prey phenotype), influences the expression of these individual trait differences. We then test these predictions using an array of experiments on different species of anuran larvae growing at different rates in the presence and absence of predators. Last, we discuss ecological and evolutionary implications of our findings.

\section{Predictions \\ Background}

Because growth rate typically scales with body size (Peters 1983), changes in size variation are inherent to the growth process. In this case, changes in size variation result strictly from effects due to size alone; we term this first class of factors "size-dependent" factors (Peacor and Pfister 2006, Peacor et al. 2007). For example, if the growth rate of larger individuals exceeds that of smaller individuals, then size variation will increase (termed "growth depensation"; Ricker 1958). The reverse will be the case if growth of smaller individuals exceeds that of larger. A simple rule predicts that the relative size variation (e.g., as measured by the coefficient of variation) will change in proportion to the change in mean growth rate of the population (Peacor et al. 2007).

Differences in traits other than size (e.g., behavior, morphology, physiology) (Fuiman and Cowan 2003) that have a genetic basis (Conover and Munch 2002, Sanford et al. 2003) or are learned (Dukas and Bernays 2000) can also generate differences in growth rate and hence affect size variation. We denote this broad group of factors as "size-independent" factors to distinguish them from the size-dependent (scaling) factors. For example, traits associated with foraging ability or "boldness" (Coleman and Wilson 1998, Sih et al. 2004) can lead to differential performance of individuals and thereby influence cohort size variation. Size-independent traits that are persistent within individuals through time have been described quantitatively as "growth autocorrelation" (Pfister and Stevens 2002), "residual autocorrelation" (Fujiwara et al. 2004), and "memory" (DeAngelis et al. 1993).

This division of factors that affect growth into sizedependent and size-independent components is analogous to the division of density-dependent and densityindependent factors in population growth equations. For example, in the general per capita population growth equation

$$
\left(\frac{1}{N}\right) \frac{d N}{d t}=r f(N)
$$

the population growth rate is described by a densityindependent coefficient, $r$, and density-dependent contributions, $f(N)$. Similar distinctions can be made in the growth equations for individuals. It should be noted that there will be a positive feedback between size-independent and size-dependent factors in the growth model; an individual that grows faster due to size-independent factors will be larger, and this in turn could confer an additional size-dependent (i.e., scaling) advantage. The fundamental difference in these two broad classes of factors is explicit when represented in growth-rate equations; to describe size-independent factors, the magnitude of parameters in growth-rate equations varies among individuals, whereas they do not for sizedependent factors (Peacor et al. 2007).

In addition, stochastic effects could cause variation in conditional states (i.e., energy level or disease load) that could affect individual growth over long periods of time and therefore cohort size variation (Ludsin and DeVries 1997). Or, resources or other factors that affect growth may be heterogeneous, which could lead to persistent unequal resource use or assimilation among individuals, and therefore affect size variation (Pfister and Peacor 2003). Whereas these factors also could be categorized in the broad class of size-independent factors, we focus on size-independent factors that arise from individual trait differences.

It is important to note that both size-dependent and size-independent factors influence variation by affecting the relative performance of different individuals. This is in contrast to an environmental factor that affects growth of all individuals by the same proportionality constant. Such a factor simply affects the rate at which mean size and variation in size change, but not their relationship to each other (Fig. 1). An experiment may show that a factor affects size variation, but if there was also a large effect on mean size, it is difficult to determine if the change in size variation is indicative of relative performance differences (Fig. 1). In this paper, we focus on size variation that arises from modifications in the relative performance of individuals. 


\section{Prediction of context-dependent predator effect}

Predator presence can potentially affect size variation through both size-dependent and size-independent factors. First, although the scaling of growth rate with size is typically thought of in terms of physiological processes (with growth scaling in the range of the 0.75 power of mass [Peters 1983]), predators can affect this scaling if predation risk is size-dependent. For example, in anuran larvae, predation risk due to larval dragonflies decreases with size, and the behavioral modifications that reduce predation rate (e.g., reduction in activity level) correspondingly decrease with prey size (Eklöv and Werner 2000; M. E. Fraker and S. D. Peacor, unpublished data). Thus predators can cause an increase in the effective scaling of growth rate with size, and this will act to increase cohort size variation (larger, more active individuals will grow proportionately more than smaller, less active individuals). Conversely, if predation risk increases as a function of size, predators may cause a decrease in the scaling of growth rate with size, which will cause a decrease in size variation.

The effect of predator presence on size variation mediated through size-independent factors is less clear. At first glance, we might assume that predator presence would lead to an increase in individual differences, with some individuals responding more strongly to the presence of the predator than others. But an equally plausible hypothesis is that signals of predator presence provide reliable information on risk that causes all individuals to behave more uniformly. For example, some individuals may be bolder than others at very low predation risk, but when predation risk is greater, all individuals may respond strongly and forage at a more similar (reduced) rate. Thus the effect of predators on individual differences that affect growth could be positive or negative. Unfortunately, there is virtually no information in the literature on how predators affect expression of individual differences in prey phenotype. Consequently, we first performed an experiment to evaluate this question in order to refine our predictions.

Resource level also can affect size variation (Jobling 1983, Uchmański 1985). Indeed, we have shown that size variation of wood frog tadpoles increases with reduced resource levels, causing slower growth (Peacor and Pfister 2006; see also Wilbur and Collins 1973). Model analyses, in which mean growth and size variation were simulated, showed that at high resource levels (high growth rate) size-variation decreased. Models based on size-dependent factors explained the pattern between mean size and size variation well, whereas even small contributions from size-independent factors caused model results to deviate from observed patterns (Peacor and Pfister 2006). In contrast, at low resource levels (low growth rate), size variation was much higher at the same mean size than with high resource levels. In fact, size variation increased as a function of mean size. Therefore, at low resource levels, variation in individual growth rates increased. Model analyses indicated that a

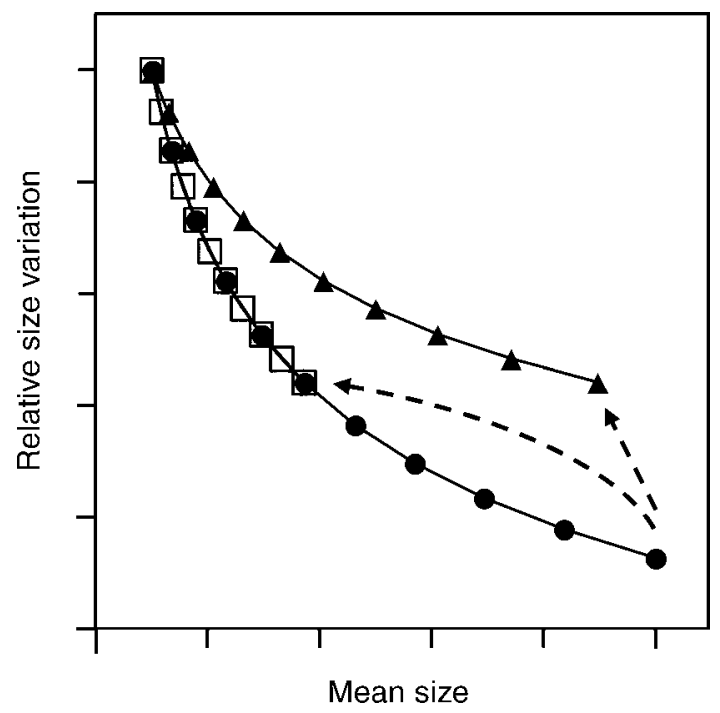

FIG. 1. Illustration of treatment effects that lead to a change in size variation with and without a change in relative individual performance. The relative size variation (e.g., as measured by the coefficient of variation) is plotted as a function of mean size (note that units are arbitrary). In the base case (solid circles), size variation decreases with growth. Two cases are presented that lead to an increase in size variation relative to the base case: reduced size variation with unmodified performance (open squares) and reduced size variation with modified performance (solid triangles). The curved arrow indicates a putative treatment that reduced growth rate and size variation such that the variation-mean size trajectory overlaps that of the unmanipulated base case, whereas the straight arrow indicates a treatment in which size variation changes and is a very different value at the same mean size as the base case. This is an important distinction when making inferences from size variation. In the former, the manipulation changed the rates of change in size and size variation but did not change how individuals perform relative to each other, whereas in the latter case both the rates of change and relative performance of individuals were affected.

reduction in resource level could cause this effect by influencing either size-dependent or size-independent factors. These results suggest that if size-independent factors are important, they will have a relatively larger influence on size variation at low resource levels. This is consistent with previous studies arguing that trait variation in the ability to acquire (find and garner) resources will have little effect on variation in growth if resources are not limiting (since all individuals will acquire sufficient resources), but will influence variation in growth when resources are scarce (Uchmański 1985, Lomnicki 1988).

Integrating the ideas that resource level will affect the relative contribution of size-dependent and size-independent factors, and that predator presence may affect each factor differently, we predict that a predator's effect on size variation may vary as a function of resource level (i.e., context-dependent predator effect; Fig. 2). At high growth rates (high resource levels) we predict that the effects of predator presence on size 


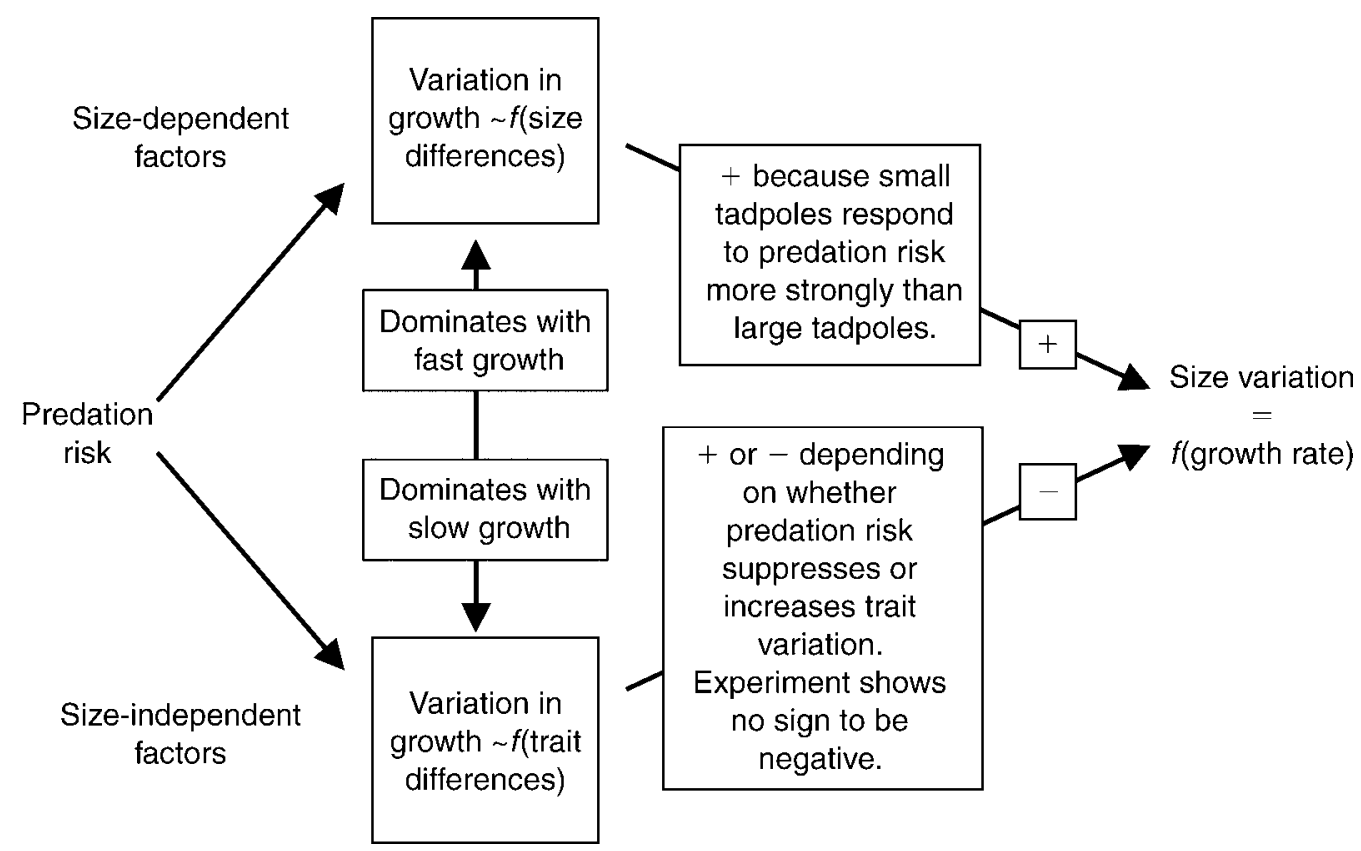

FIG. 2. Conceptual diagram of the nonlethal effect of a larval dragonfly predator on tadpole cohort size variation. Both sizedependent and size-independent factors can affect size variation. The former will dominate at fast growth rates (high resource levels), and therefore variation in growth is largely a function of size. The latter will dominate at slow growth rates (low resource levels), and therefore variation is largely a function of trait differences (other than size). The predator influence on size-dependent factors will be positive (note that this sign could be negative for other predator-prey interactions). Our experiment indicates that the predator's influence on size-independent factors is negative. Therefore, the larval dragonfly predator's effect on size variation is a function of tadpole growth rate.

variation will be positive due to the positive influence of the predator on size-dependent factors (Fig. 2). At low growth rates (low resource levels), we predict that the effects of size-independent factors will be more pronounced. Predator presence could increase or suppress the expression of size-independent factors, which would either augment or oppose the positive effect of the predator due to size-dependent factors. In the latter case, the predator could have a net negative effect on relative size variation. Thus effects of the presence of a predator on size variation could be positive or negative depending on growth rate (resource level). The predictions at low growth rates turn on both whether the presence of predators affects size-independent factors and in which direction.

\section{Predator Effects on Size-Independent Factors}

\section{Experimental methods}

We designed the following experiment to determine if among-individual differences in traits (other than size) affect the development of size variation in bullfrog tadpoles (Rana catesbeiana), and if predator presence influences expression of these trait differences. The basic approach was to quantify the intermixing (i.e., overlap in distributions) of two initially distinct size classes raised together, and determine how predator presence affected this intermixing (Fig. 3). We expect that some degree of intermixing will occur due to stochastic processes or resource heterogeneity (see Predictions: Prediction of context-dependent predator effect). However, if growth is strictly a size-dependent process, then the predator will have little or no effect on the degree of intermixing of the two size classes. That is, the fraction of overlap in the distributions of the size classes will remain unchanged because the predator will not affect the rank order of individuals. However, if individual trait differences cause significantly differential growth rates, this could lead to intermixing of the two classes, as faster-growing individuals from the smaller size class could achieve larger sizes than slower-growing individuals from the larger size class. If the predator affects expression of these trait differences, it will consequently have an effect on the degree of intermixing due to sizeindependent factors. This design tests for an effect of size-independent factors above and beyond any effect they may have had on the initial distribution. That is, size-independent factors, and/or size-dependent effects in combination with stochastic effects, may be responsible for the initial generation of size variation. We examine whether size-independent factors have any additional effects after further growth.

The study was performed at the University of Michigan's E. S. George Reserve experimental pond site in southern Michigan. Experiments were conducted 
b) Final distributions, with size-dependent factors
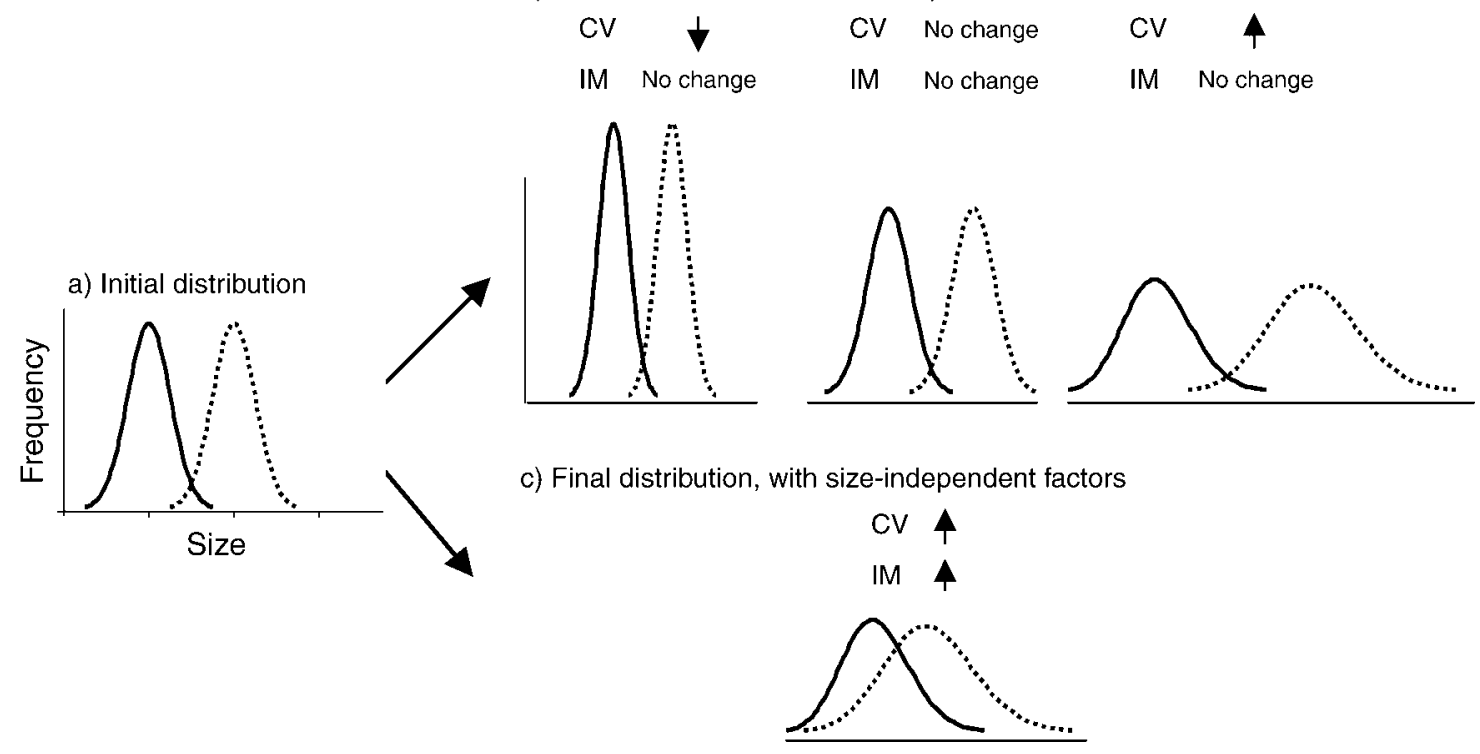

FIG. 3. Hypothetical distributions of the small- and large-group size classes in the experiment: (a) initial size distributions, and (b) expected final size distributions due to size-dependent factors; and (c) expected distributions due to size-independent factors. If variation in growth is influenced by size-dependent factors, then relative size variation (e.g., as measured by CV) of both groups may decrease, remain unchanged, or increase, depending on size-growth scaling relationships. In all cases, however, there will be no change in the rank order of individuals, and therefore the level of intermixing (IM) will be unaffected. In contrast, if variation in growth is influenced by size-independent factors, there will be changes in rank order of individual within and between groups, which will increase intermixing in addition to relative size variation within groups. Therefore the level of intermixing is an index of the influence of size-independent effects. Note that this is idealized for clarity; we also expect stochastic effects to lead to intermixing, and therefore size-independent effects would be expressed as an increase in intermixing above this effect.

in cylindrical $300-\mathrm{L}$ wading pools filled with well water and covered with $60 \%$ shade cloth to deter colonization by aquatic insects and other frogs. Each pool contained three predator cages constructed from slotted plastic drainpipe $(11 \mathrm{~cm}$ diameter $\times 10.5 \mathrm{~cm}$ length $)$ with ends enclosed by fiberglass window screening. On 1 August 2003, we added $100 \mathrm{~g}$ of dry oak leaves (Quercus sp.) to each pool to provide physical complexity, $2 \mathrm{~g}$ of rabbit chow to provide initial nutrients for tadpoles and periphyton growth, and aliquots of phytoplankton and periphyton collected from a nearby pond.

Tadpoles for the experiment originated from four egg masses, collected from ponds at the Saline Michigan Department of Natural Resources site. Hatchling tadpoles were mixed and raised in three separate wading pools and were fed rabbit chow. On 11 August, all tadpoles were combined and sorted into "small group" and "large group" size classes. In order to differentiate the small- and large-group tadpoles, tadpoles from the large group were anesthetized (using diluted clove oil) and marked subcutaneously with red fluorescent elastomer dye (Northwest Marine Technology, Shaw Island, Washington, USA) on 12 August and 13 August. Large tadpoles were chosen because they were easier to mark. On 14 August we again scanned the populations and removed the largest tadpoles from the small group, and the smallest tadpoles from the large group to assure that there was little overlap in size between the two groups. A sample from these groups $(N=150)$ was weighed, and the results indicated that there was very little overlap between the two groups.

From these two groups of tadpoles, 30 small-group unmarked tadpoles (mass $=77.8 \pm 8.6 \mathrm{mg}$; mean $\pm \mathrm{SD}$ ) and 30 large-group marked tadpoles (mass $=118.1 \pm$ $18.3 \mathrm{mg}$ ) were added to each wading pool on 14 August. Resources were added through the experiment by adding $6.7 \mathrm{~g}$ ground rabbit chow per pool per week (divided into three equal rations per week). In half of the pools, one larval Anax (dragonfly) predator was added to each of the three cages. Each Anax was fed 3-5 bullfrog tadpoles (totaling 250-300 mg), three times per week (as in, e.g., Peacor [2002]). We did not quantify the effect of the predator on tadpole behavior, but observations during feeding indicated that tadpoles responded by reducing activity and spending more time on pool bottoms in the presence of a predator, as observed in numerous other experiments (e.g., Peacor 2002). There were five replicates for both treatments (i.e., predator and no predator).

On 3 September, 19 days after initiation of the experiment, tadpoles were dipnetted out of the pools, sorted into marked and unmarked groups, and weighed individually. If a large-group tadpole was unaccounted for (i.e., when they were not all recovered), it was not possible to know if it died or lost its mark. It would therefore be possible to assign a large-group tadpole 
(with a missing mark) to the small-group. In order to be conservative, if $X$ large tadpoles were unaccounted for in a pool, we discarded the largest $X$ individuals from the small group in the analysis (except when analyzing total tadpole survival). The total number of tadpoles discarded in this manner was 12 (6 in both predator treatments), or on average 1.2 per pool (ranging from 0 to 3 ), and this procedure had little effect on the results.

We quantified the magnitude of the intermixing of the small- and large-group tadpoles for each pool using a "fraction intermixed" (FI) metric; i.e., the average degree to which small-group individuals merged into the large-group distribution. FI was computed as the average proportion of large-group individuals that small-group individuals were larger than as follows:

$$
\mathrm{FI}=\frac{1}{n_{\mathrm{s}} n_{1}} \sum_{i=1}^{n_{\mathrm{s}}} N_{i}
$$

where $n_{\mathrm{s}}$ and $n_{1}$ are the final number of small- and largegroup individuals, respectively. $N_{i}$ is the number of large-group individuals that were smaller than a particular small-group individual, $i$. Thus FI is computed as follows: (1) for each small-group individual, count the number of large-group individuals that are smaller, (2) sum this value over all small-group individuals, and (3) divide this sum by the product $n_{\mathrm{s}} n_{1}$, which is the total number of combinations of small-group and large-group individuals. The FI metric provides an intuitive picture of the degree of intermixing; it is equivalent to the average proportion of large (small) group individuals that average small-group individuals are larger (smaller) than. If there is no intermixing, FI is zero, whereas if there is complete intermixing, FI is 0.5 (i.e., small-group individuals are on average larger than half the largegroup individuals). Note that FI is the probability that a small-group individual is larger than a large-group individual. The initial intermixing was nearly absent, as intended, with a value of $\mathrm{FI}=0.002$

We used Hotelling's $T^{2}$ test to examine the effect of the predator on tadpole growth (mass increase), using the small- and large-group tadpoles as two dependent variables. Where significant, we examined each group separately with a $t$ test. The same procedure was used to examine the effect of the predator on $\mathrm{CV}$ in size. We also used a $t$ test to examine the effect of predator presence on survival (of all tadpoles in a mesocosm), the ratio of growth of small- and large-group tadpoles, and the degree of intermixing (FI).

One method to distinguish size-dependent and sizeindependent effects is to mark and follow the growth of individuals (Pfister and Stevens 2003). Although a powerful approach, it is not always practical to collect data repeatedly on the same individual. In our case, time and resource constraints did not permit distinctive markings of individuals. However, our methodology allowed us to determine if size-independent factors were important.

\section{Results and interpretation}

Survival of the tadpoles was very high, with only 11 (out of 600) tadpoles unaccounted for. Predator presence did not affect tadpole survival $(P=0.59)$. Therefore mortality did not confound predator effects on the mean and on variation in size.

Small-group and large-group tadpoles grew to $192 \pm$ $54 \mathrm{~g}$ (mean $\pm \mathrm{SD})$ and $266 \pm 72 \mathrm{~g}$, respectively (Fig. 4a) There was no effect of predator presence on mean growth (Hotelling's $T^{2}$ test, $F_{2,7}=0.94, P=0.44$ ). The fact that predator presence in this experiment had no effect on mean tadpole growth (while still affecting behavior) indicates that resource levels were relatively low and limited growth. We have shown both theoretically, and empirically in a number of experiments, that a predator-induced reduction in tadpole foraging effort will have a strong negative effect on prey growth at high resource levels, grading to no effect at low resources (Peacor and Werner 2000, 2004).

The predator had a strong negative effect on the relative size variation $(\mathrm{CV})$ within size groups (Fig. 4b, Hotelling's $T^{2}$ test, $\left.F_{2,7}=29.0, P<0.001\right)$. This negative effect was significant for both the small- and large-group tadpoles ( $P<0.001$ for both groups). There also was a substantial amount of intermixing (Fig. 4c), with FI higher in the absence $(0.30 \pm 0.013)$ than in the presence $(0.13 \pm 0.013)$ of the predator $(P=0.0012)$. The predator effect on variation was further realized in the ratio of the largest to smallest tadpole in a pool (Fig. $4 d)$, which was 2.4-fold smaller in the presence of a predator $(P=0.01)$.

The predator effect on size distributions and intermixing are clearly evident in a histogram of the size distributions (Fig. 5). In the presence of a predator, the distribution is much narrower, with fewer unusually large individuals for both the small- and large-group tadpoles, and there is a marked decrease in intermixing between small- and large group size classes. Note that the median size of the small- and large-group distributions are nearly identical in predator presence and absence (0.822 and 0.829 for small-group tadpoles in predator presence and absence, respectively, and 1.177 and 1.174 for large-group tadpoles in predator presence and absence, respectively; Fig. 5). This indicates that whereas predator presence had a large effect on the relative performance of tadpoles (variation), it had no effect on the mean performance of the two groups as a whole.

Two lines of evidence indicate that the predator affected size variation by suppressing size-independent factors. First, if predator presence reduced size variation via size-dependent factors, then predator presence would also reduce the difference in mean sizes between the small and large groups, which was not observed. Further, predator presence is predicted to increase, not suppress, size variation resulting from size-dependent factors (see Predictions: Prediction of context-dependent predator effect). In contrast, if predator presence led to the observed reduction in size variation via size- 

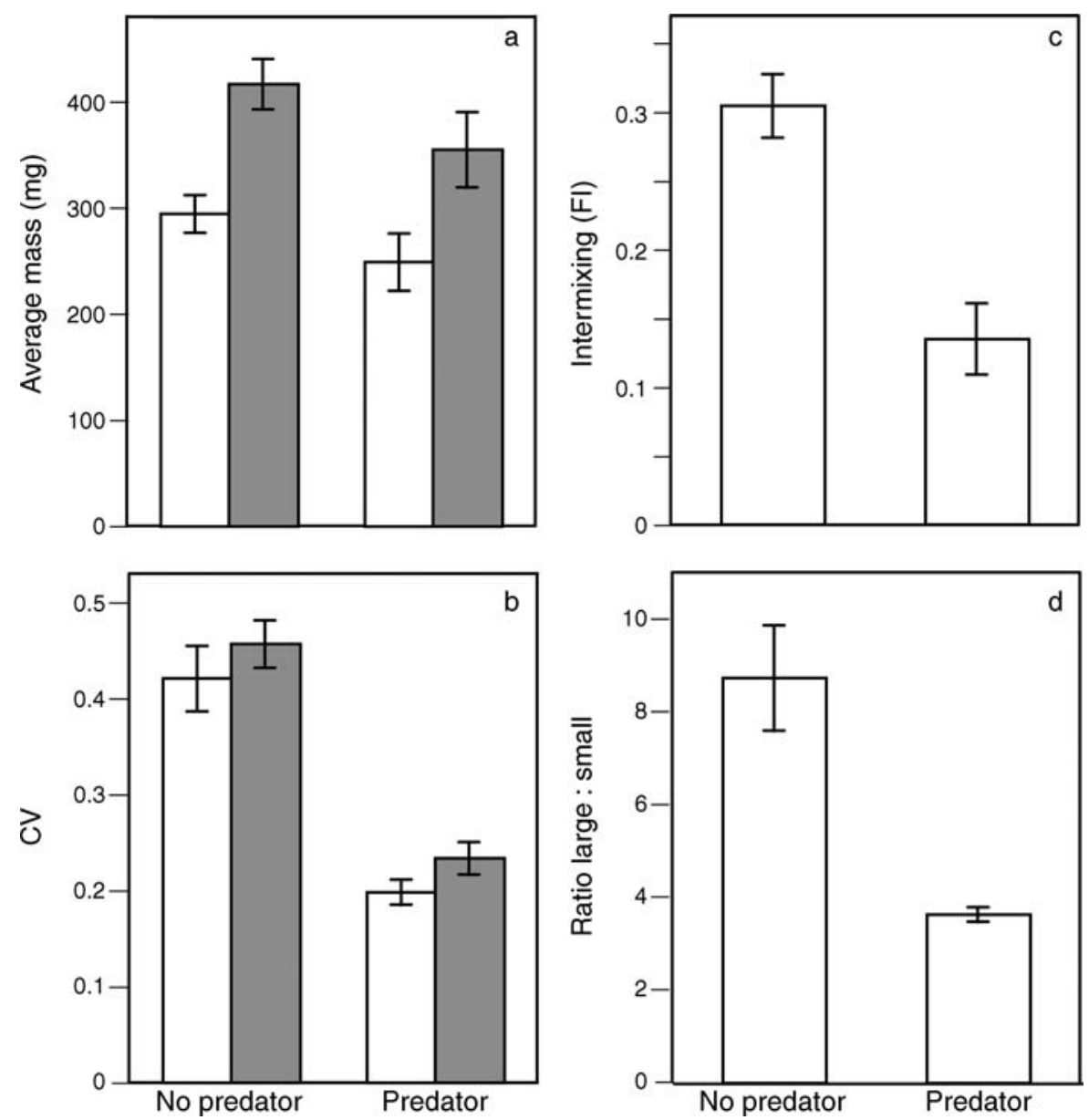

FIG. 4. Effect (mean \pm SE) of caged Anax predators on bullfrog tadpoles: (a) average mass, (b) relative size variation (CV), (c) fraction intermixing metric (FI), and (d) ratio of the largest and smallest tadpoles. In (a) and (b) the responses of small-group (white) and large-group (gray) tadpoles are both shown, whereas responses in (c) and (d) are based on tadpoles from both groups.

independent effects alone, differential effects on individual growth within the groups responsible for the decreased size variation should cancel out and not affect the mean growth of each group, as observed.

Second, only size-independent factors, not size-dependent factors, can account for the simultaneous increase in $\mathrm{CV}$ of both the small- and large-group tadpoles and the decreased level of intermixing seen when predators are present (Fig. 3; see Predictions: Prediction of contextdependent predator effects). We cannot envision how the absence of the predator would increase the contribution of stochastic or history effects on intermixing. In addition an increase in history effects (e.g., large tadpoles were placed in clove oil and marked, and then placed in different pools the day before the experiment) would not be associated with an increase in $\mathrm{CV}$ in both small and large groups. Therefore this experiment indicates a strong contribution of size-independent factors on size variation, and that predator presence reduces this contribution.

\section{Tests of Predictions}

These experimental results inform our predictions of the influence of the predator on size variation. Recall that we predicted that predator presence will have a positive effect on size variation through size-dependent effects at high growth rates. As growth rates decrease, however, we predicted size-independent effects would become increasingly important, and the experiment indicated that predator presence suppresses variation due to this factor. Consequently, we predicted that at fast growth rates, the predator would have a positive effect on the CV, and as growth rate decreases, the positive effect will be reduced and potentially even be reversed.

In order to test these predictions, we reviewed a number of our experiments (most published) where we could quantify cohort size variation. Some of these experiments were not designed specifically to explore the effect of predators on individual variation per se (see Appendix for methods), but rather were conducted to 


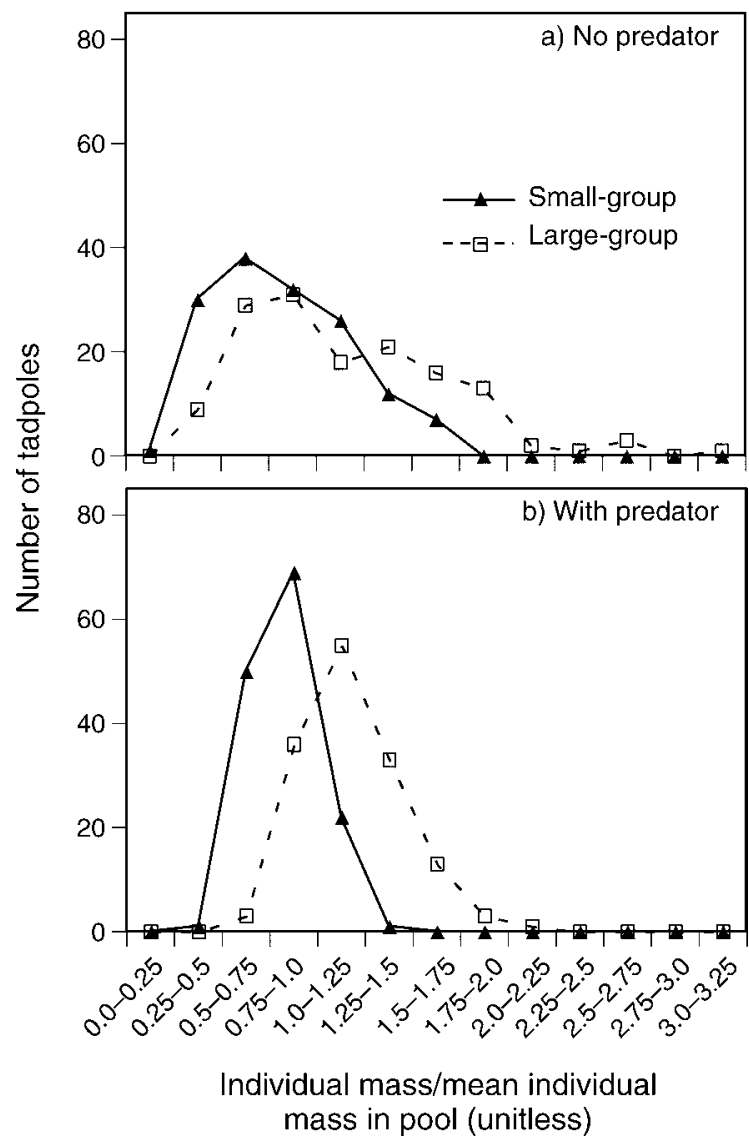

FIG. 5. Size distribution of small-group and large-group bullfrog tadpoles in (a) the absence and (b) the presence of caged Anax predators. Each panel contains all surviving tadpoles from five replicates. The size of each individual tadpole was normalized to the mean of all tadpoles within its pool.

examine the nonlethal effect of predators on tadpole behavior, growth, and community organization. However, taken as a whole, these experiments present a coherent pattern of the influence of predator presence on individual variation for two additional species of anuran larvae over a range of different growth rates. In order to insure that patterns in variation were due to changes in individual performance (for the reasons laid out in Fig. 1 ), we included only data from experiments where mean mass in predator presence and absence was approximately equal (predator effect on mean mass $<10 \%$, with two exceptions in which inclusion is conservative; see Appendix). This constraint does not indicate that the predator had little effect on tadpole behavior. Rather, in all cases, the predator induced a reduction in the foraging rate of the tadpoles. As noted earlier, if the predator induces a reduction in the foraging rate of all tadpoles in a population, theory and empirical research indicate that the effect on mean tadpole growth rate can be negative, small, or even positive, depending on resource supply rates and the duration of the experiment (Peacor and Werner 2000, 2004, Peacor 2002).
We have data on two species of ranid anurans (the wood frog, Rana sylvatica, and the green frog, $R$. clamitans) encompassing a range of growth conditions that can be used to test these predictions. Experiments were all performed in either $300-\mathrm{L}$ or $1100-\mathrm{L}$ mesocosms. To mimic conditions of natural ponds, the mesocosms were inoculated with phytoplankton, periphyton, and zooplankton. Dry leaves (predominantly Quercus sp.) were added to provide physical complexity, serve as a resource (detritus), and act as a substrate for periphyton. In all cases, the predators were caged dragonfly larvae (Anax spp.) that were fed the target tadpole species (see Appendix for methods). To quantify the predator effect on size variation, we calculated the relative change in $\mathrm{CV}$ of tadpoles in predator presence (i.e., $[\mathrm{CV}$ with predator absent $-\mathrm{CV}$ with predator present $] / \mathrm{CV}$ with predator absent). We examined this effect as a function of mean growth rate, calculated as $\ln$ (final mass/initial mass)/number of days, in predator presence and absence.

First, consider the wood frog data taken from three experiments (Appendix). In each of the experiments, there was a trend of increasingly positive effects of the predator on $\mathrm{CV}$ in treatments with higher growth rates (Fig. 6a). Moreover, when all experiments were combined, the overall relationship was strong and significant (Fig. 6a; linear regression, $F_{1,7}=19.0, P=0.003$ ). Note that the negative effects of the predator on the $\mathrm{CV}$ at the lowest growth rate, and the positive effects at the higher growth rates, were significantly different from zero (see Appendix for statistics).

Data from two experiments using green frog tadpoles illustrated the same pattern observed with wood frogs; means of the relative change in $\mathrm{CV}$ again ranged from negative to positive, and at increasing growth rates, the predator effect on the $\mathrm{CV}$ was increasingly positive (Fig. $6 \mathrm{~b}$; linear regression, $\left.F_{1,5}=12.4, P=0.017\right)$. The range of growth rates explored in these experiments was narrower than those with the wood frog, which may be responsible for the fact that the positive effects of the predator at the highest growth rates and the negative effects at the lowest growth rates were not significantly different from zero $(P>0.1$; Appendix $)$. However, the overall trend is significant and consistent with that of the wood frog, even with the reduced range of growth rates.

\section{Discussion}

Understanding the mechanisms responsible for generating size variation in cohorts of organisms is critical to understanding their population and evolutionary dynamics. We have argued that these mechanisms can be grouped into two broad classes, those that are sizedependent (i.e., scale with body size), and those that are size-independent (i.e., are a function of individual traits largely unrelated to size). It is useful to distinguish these two broad classes of mechanism because they can have different effects on size variation in a cohort. Further, 
these factors can interact in ways that cause the effect of a given factor on size variation to be context dependent.

We have shown that the presence of predators, which have a wide range of important effects on mean prey phenotypes (Lima 1998, Tollrain and Harvell 1999), can lead to context-dependent effects on size variation. Our experiment indicated that the presence of predators suppressed the expression of variation in individual traits. Based on this result, we predicted that the relative effect of the predator on the $\mathrm{CV}$ in size of a cohort would range from negative at low growth rates to positive at high growth rates. These predictions were borne out in examining results of a series of experiments on two species of anuran larvae. We interpret this trend as due to the different factors dominating at the different growth rates. At high growth rates, size-dependent factors dominate the effects on size variation. Because vulnerability of the tadpoles declines with size (e.g., Eklöv and Werner 2000), the predator acts to increase size-dependent differences in growth (the scaling factor) and therefore leads to an increase in the $\mathrm{CV}$ at high growth rates. In contrast, at low growth rates when the tadpoles are stressed due to resource limitation, sizeindependent factors (based on traits such as activity, assimilation rates, etc.) become relatively more important. Because predator presence suppresses individual variation in these traits, it suppresses cohort size variation at low resource levels. Thus the relative magnitude of size-independent and size-dependent effects due to the predator can change with environmental context and, in cases, reverse the effect of the predator on cohort size variation relative to controls. These effects are important as they have implications for the development of cohort size variation and the transparency of traits to natural selection.

Our results are likely quite general. Given the wide range of systems where predators exhibit size selection of prey, it is likely that size-dependent effects (that influence the scaling factor) will have a strong impact on cohort CV in many systems. For example, if predators select larger individuals, then larger individuals will exhibit stronger antipredator responses, which come at the cost of reduced growth rate. Predator presence will therefore act to decrease size variation by reducing the relative scaling advantages of larger sizes. However, if predators select smaller individuals, which then exhibit stronger antipredator responses (e.g., as with anuran larvae), this will increase the relative scaling advantage of larger individuals. Ziemba et al. (2000) have demonstrated this effect in cannibalistic tiger salamander larvae, in which small larvae become less active (forage less) in the presence of larger individuals, thus increasing cohort size variation. Note that the net predator effect on size variation will be a function of both these nonlethal effects and size-selective predation.

Predators, however, also have a wide range of effects on phenotypic traits of prey that are largely size independent, e.g., activity levels, boldness, body form,

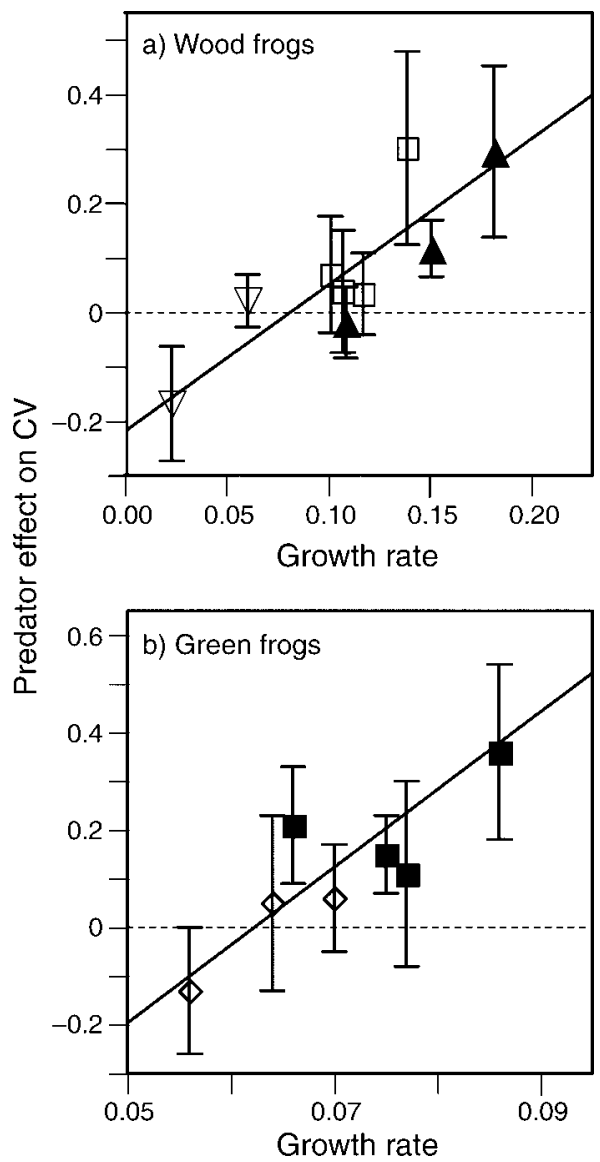

FIg. 6. The relative effect of caged Anax predators on CV $\left(\left[\mathrm{CV}_{\mathrm{PA}}-\mathrm{CV}_{\mathrm{PP}}\right] / \mathrm{CV}_{\mathrm{PA}}\right)$ of tadpoles as a function of growth rate (mean in predator presence [PP] and absence [PA]). Error bars are propagated error from the standard error of the means in predator presence and absence. (a) Wood frog data from three experiments. (b) Green frog data from two experiments. Different experiments are shown by different symbols. The lines are the best fit through all data points, meant as a visual guide. Note the difference in scale in the two figures.

and metabolic efficiencies (McPeek et al. 2001, Relyea and Auld 2005; reviewed in Lima 1998, Tollrain and Harvell 1999). Although there is a large body of literature indicating the strong effect of predators on the mean value of these traits, little is known about how predators affect individual variation in expression of these traits. We are aware of only one study that indicates that predators affect variation in traits (i.e., in traits other than size). Coleman and Wilson (1998) showed that variation in time spent alone and time spent in open water in pumpkinseed sunfish was higher in the presence of bass predators. As we have shown here, the presence of a predator also can suppress variation in expression of phenotypic traits, which has a very important impact on the $\mathrm{CV}$ in prey size, especially at low resource levels. This in turn can have large effects on population dynamics and the potential for selection. 
It is unclear from our experiment which phenotypic trait or traits were responsible for the individual size variation. It is possible that resource specialization is responsible (e.g., Bolnick et al. 2003); for example individuals may have different preferences for periphyton growing on pool walls relative to the artificial resources that primarily settle to the pool floor. Alternatively, individual differences in activity level, which have been shown to be positively related to resource acquisition and growth across species (e.g., Relyea and Werner 1999), may be the trait responsible. In a laboratory study, we have observed that individual differences in activity level persisted in bullfrog tadpoles for the duration of a two-week experiment (S. D. Peacor, unpublished data). Similarly, a large and growing literature documents individual "personality" differences (behavioral syndromes) in animals (Wilson 1998, Sih et al. 2004). Often the behavioral traits examined in such studies are associated with risk taking or boldness. These traits have clear parallels with activity level, which affects both resource acquisition rate and predation risk.

A number of theoretical studies show that incorporating individual variation in population models can impact population dynamics (Lomnicki 1988, Conner and White 1999, Pfister and Stevens 2003). Using an individually based model, Grimm and Uchmański (2002; see also Uchmański 1999) found that the stability of the model population was strongly influenced by variation in size and growth rate. Fox (2005) showed that individual variation in demographic variables such as size can be as important in reducing extinction risk of small populations as increasing population density itself. De Roos et al. (2003) found that incorporating sizedependent predation and growth had profound effects in size-structured models. Increased variation also can have dynamical consequences by expanding resource axes that mediate cannibalistic and competitive interactions (Van Valen 1965, Roughgarden 1974, Bolnick et al. 2003). Taken as a whole, these studies indicate that incorporating individual variation can have substantial effects on population dynamics, and therefore factors such as predator presence that affect this variation could be very important in influencing dynamics.

More specifically, our results indicate that the presence of a predator could have large population consequences to larval anurans through effects on individual variation in size. It is well known that threshold sizes are required to initiate metamorphosis in anurans (Wilbur and Collins 1973), and that pond drying can intercede during development and cause wholesale mortality of anuran cohorts. Consider the potentially strong effects on both population dynamics and evolution in these anurans if drying intercedes when cohort size structure is such that some fraction of the population can metamorphose and escape the pond. Our results indicate that even if the presence of a predator had no effect on mean growth rate, it could have an enormous effect on the fraction of the population that achieves the threshold required for metamorphosis when drying occurred. This effect then could influence population dynamics and selection, possibly more strongly than the effects of mortality due to predation.

Individual variation, of course, is the grist of the evolutionary mill, and the fact that predators can have important effects on expression of this variation has potentially important implications for evolutionary dynamics. The response of a trait to selection is a function of its heritability, and phenotypic responses to the presence of a predator can change this heritability by altering the proportion of phenotypic variation explained by genetic variation (e.g., Fordyce 2006). For example, if a trait such as boldness or activity level exhibits large variation in the absence of a predator, and one extreme is favored by local conditions, we would expect rapid evolutionary change for the selected phenotype. However, if the presence of the predator suppresses such variation, we would expect much weaker responses to selection on that trait. That is, predators can affect the penetrance of alleles by weakening the relationship between genetic variation in behavioral type and growth, and therefore the effectiveness of selection on the trait. Presence and absence of the predator thus can cause temporal variation in the heritability of traits (Fordyce 2006). Alternatively, if the predator suppresses variation that is strictly environmentally induced, this could increase exposure of existing genetic variation and accelerate the response to evolution. Effectively, the predator can mask (or expose) variation to natural selection as well as be a strong agent of natural selection itself.

The responses to predators also can have important consequences for evolutionary responses to interactions with other species. For example, high activity rates or other traits enhancing growth rates can be important in competitive interactions among species of anuran larvae (Werner 1992). If a competing species is not as vulnerable to the predator (e.g., is toxic or larger) or does not respond in a parallel fashion, then plasticity in responses by the focal species to a predator presents a "moving target" to natural selection of adaptive responses of the competing species. As Fordyce (2006) points out, such plasticity in the focal species creates a variable biotic environment for the competitor, which in turn may favor the evolution of plasticity in the competitor. This variable biotic environment reduces the potential for trait-by-trait pairwise coevolution between interacting species. Predator-induced changes in individual variation also may have evolutionary implications through effects on frequency-dependent interactions. For example, variation in traits leading to size variation can affect intraspecific competition, with higher competitive effects on phenotypically intermediate individuals. This variation has implications for a suite of evolutionary processes, including disruptive selection, niche expansion, and adaptive radiation 
(Bolnick 2001). Clearly, if predator presence affects the level of individual variation, it will in turn affect such evolutionary processes.

In conclusion, we have shown that the presence of predators interacting with resource levels can cause context-dependent effects on variation in size among cohorts of anuran larvae. By separating the effects into those that scale with size vs. those that are independent of size, we were able to understand how this context dependence develops and predict the direction of the effects. This perspective should help in understanding the net consequences of the presence of predators on prey populations. The history of predator-prey theory has focused on the numerical impact of the predator on prey populations through direct consumption of prey. More recently it has become apparent that the nonlethal effects of a predator (via predator-induced changes in prey phenotype) can in many cases have equivalently strong effects on the prey (or indirectly on species with which the prey interacts; e.g., Werner and Peacor 2003). Our results indicate a further mechanism whereby this nonlethal effect of predators can be manifest and suggest that the mere presence of predators can have important effects on population dynamics and evolution of their prey. Given the critical importance of variation in size in cohorts during their ontogeny to both population dynamics and natural selection, these mechanisms deserve further scrutiny.

\section{ACKNOWLEDGMENTS}

We thank Zack Schulman for assistance conducting the experiment and Mara Zimmerman for assistance with the statistical analysis. We thank Dan Bolnick, Cathy Pfister, and the Werner lab reading group, as well as two anonymous reviewers, for helpful comments on the manuscript. We thank Allan Sutton for permission to collect specimens at the Michigan Department of Natural Resources Saline Pond Facility. This research was supported by NSF grants DEB9615523 and DEB-0089809 to E. Werner and S. Peacor and support from the Michigan Agricultural Experimental Station to SDP. This is Great Lakes Environmental Research Laboratory contribution number 1417.

\section{Literature Cited}

Bolnick, D. I. 2001. Intraspecific competition favours niche width expansion in Drosophila melanogaster. Nature 410: 463-466.

Bolnick, D. I., R. Svanback, J. A. Fordyce, L. H. Yang, J. M. Davis, C. D. Hulsey, and M. L. Forister. 2003. The ecology of individuals: incidence and implications of individual specialization. American Naturalist 161:1-28.

Bonner, J. T. 2006. Why size matters: from bacteria to blue whales. Princeton University Press, Princeton, New Jersey, USA.

Coleman, K., and D. S. Wilson. 1998. Shyness and boldness in pumpkinseed sunfish: individual differences are contextspecific. Animal Behaviour 56:927-936.

Conner, M. M., and G. C. White. 1999. Effects of individual heterogeneity in estimating the persistence of small populations. Natural Resource Modeling 12:109-127.

Conover, D. O., and S. B. Munch. 2002. Sustaining fisheries yields over evolutionary time scales. Science 297:94-96.

DeAngelis, D. L., K. A. Rose, L. B. Crowder, E. A. Marschall, and D. Lika. 1993. Fish cohort dynamics: application of complementary modeling approaches. American Naturalist 142:604-622.

De Roos, A. M., L. Persson, and E. Mccauley. 2003. The influence of size-dependent life-history traits on the structure and dynamics of populations and communities. Ecology Letters 6:473-487.

Dukas, R., and E. A. Bernays. 2000. Learning improves growth rate in grasshoppers. Proceedings of the National Academy of Sciences (USA) 97:2637-2640.

Eklöv, P., and E. E. Werner. 2000. Multiple predator effects on size-dependent behavior and mortality of two species of anuran larvae. Oikos 88:250-258.

Fordyce, J. A. 2006. The evolutionary consequences of ecological interactions mediated through phenotypic plasticity. Journal of Experimental Biology 209:2377-2383.

Fox, G. A. 2005. Extinction risk of heterogeneous populations. Ecology 86:1191-1198.

Fuiman, L. A., and J. H. Cowan. 2003. Behavior and recruitment success in fish larvae: repeatability and covariation of survival skills. Ecology 84:53-67.

Fujiwara, M., B. E. Kendall, and R. M. Nisbet. 2004. Growth autocorrelation and animal size variation. Ecology Letters 7: 106-113.

Gardmark, A., U. Dieckmann, and P. Lundberg. 2003. Lifehistory evolution in harvested populations: the role of natural predation. Evolutionary Ecology Research 5:239-257.

Grimm, V., and J. Uchmański. 2002. Individual variability and population regulation: a model of the significance of withingeneration density dependence. Oecologia 131:196-202.

Hutchinson, G. E. 1959. Homage to Santa Rosalia, or why are there so many kinds of animals? American Naturalist 93:145159.

Jobling, M. 1983. Effect of feeding frequency on food-intake and growth of Arctic charr, Salvelinus alpinus L. Journal of Fish Biology 23:177-185.

Lima, S. L. 1998. Stress and decision making under the risk of predation: recent developments from behavioral, reproductive, and ecological perspectives. Advances in the Study of Behavior 27:215-290.

Lomnicki, A. 1988. Population ecology of individuals. Princeton University Press, Princeton, New Jersey, USA.

Ludsin, S. A., and D. R. Devries. 1997. First-year recruitment of largemouth bass: the interdependency of early life stages. Ecological Applications 7:1024-1038.

McPeek, M. A., M. Grace, and J. M. L. Richardson. 2001. Physiological and behavioral responses to predators shape the growth/predation risk trade-off in damselflies. Ecology 82:1535-1545.

Peacor, S. D. 2002. Positive effect of predators on prey growth rate through induced modifications of prey behaviour. Ecology Letters 5:77-85.

Peacor, S. D., J. R. Bence, and C. A. Pfister. 2007. The effect of size-dependent growth and environmental factors on animal size variability. Theoretical Population Biology 71:80-94.

Peacor, S. D., and C. A. Pfister. 2006. Individual size variation in wood frog (Rana sylvatica) tadpoles: the effect of competition and model analysis of size-dependent and size-independent factors. Journal of Animal Ecology 75: 990-999.

Peacor, S. D., and E. E. Werner. 2000. Predator effects on an assemblage of consumers through induced changes in consumer foraging behavior. Ecology 81:1998-2010.

Peacor, S. D., and E. E. Werner. 2004. Context dependence of nonlethal effects of a predator on prey growth. Israel Journal of Zoology 50:139-167.

Peters, R. H. 1983. The ecological implications of body size. Cambridge University Press, Cambridge, UK.

Pfister, C. A., and S. D. Peacor. 2003. Variable performance of individuals: the role of population density and endogenously formed landscape heterogeneity. Journal of Animal Ecology $72: 725-735$. 
Pfister, C. A., and F. R. Stevens. 2002. The genesis of size variability in plants and animals. Ecology 83:59-72.

Pfister, C. A., and F. R. Stevens. 2003. Individual variation and environmental stochasticity: implications for matrix model predictions. Ecology 84:496-510.

Relyea, R. A., and J. R. Auld. 2005. Predator- and competitorinduced plasticity: how changes in foraging morphology affect phenotypic trade-offs. Ecology 86:1723-1729.

Relyea, R. A., and E. E. Werner. 1999. Quantifying the relation between predator-induced behavior and growth performance in larval anurans. Ecology 80:2117-2124.

Ricker, W. E. 1958. Handbook of computations for biological statistics of fish populations. Fisheries Research Board of Canada, Bulletin Number 119.

Roughgarden, J. 1974. Niche width: biogeographic patterns among Anolis lizard populations. American Naturalist 108: 429-442.

Sanford, E., M. S. Roth, G. C. Johns, J. P. Wares, and G. N. Somero. 2003. Local selection and latitudinal variation in a marine predator-prey interaction. Science 300:1135-1137.

Sebens, K. P. 1987. The ecology of indeterminate growth in animals. Annual Review of Ecology and Systematics 18:371407.

Sih, A., A. Bell, and J. C. Johnson. 2004. Behavioral syndromes: an ecological and evolutionary overview. Trends in Ecology and Evolution 19:372-378.

Tollrain, R., and C. D. Harvell. 1999. The ecology and evolution of inducible defenses. Princeton University Press, Princeton, New Jersey, USA.
Uchmański, J. 1985. Differentiation and frequency-distributions of body weights in plants and animals. Philosophical Transactions of the Royal Society B 310:1-75.

Uchmański, J. 1999. What promotes persistence of a single population: an individual-based model. Ecological Modelling 115:227-241.

Uchmański, J., and V. Grimm. 1996. Individual-based modelling in ecology: what makes the difference? Trends in Ecology and Evolution 11:437-441.

Van Valen, L. 1965. Morphological variation and width of ecological niche. American Naturalist 99:377-390.

Werner, E. E. 1992. Competitive interactions between wood frog and northern leopard frog larvae: the influence of size and activity. Copeia 1992:26-35.

Werner, E. E., and J. F. Gilliam. 1984. The ontogenetic niche and species interactions in size structured populations. Annual Review of Ecology and Systematics 15:393-425.

Werner, E. E., and S. D. Peacor. 2003. A review of traitmediated indirect interactions in ecological communities. Ecology 84:1083-1100.

Whitlock, M. C. 1995. Variance-induced peak shifts. Evolution 49:252-259.

Wilbur, H. M., and J. P. Collins. 1973. Ecological aspects of amphibian metamorphosis. Science 182:1305-1314.

Wilson, D. S. 1998. Adaptive individual differences within single populations. Philosophical Transactions of the Royal Society B 353:199-205.

Ziemba, R. E., M. T. Myers, and J. P. Collins. 2000. Foraging under the risk of cannibalism leads to divergence in body size among tiger salamander larvae. Oecologia 124:225-231.

\section{APPENDIX}

Methods of experiments reviewed to examine the effect of predator presence on individual size variation as a function of growth rate (Ecological Archives E088-092-A1). 